\title{
Dissolution enhancement of Capmul PG8 and Cremophor EL based Ibuprofen Self Emulsifying Drug Delivery System (SEDDS) using Response surface methodology
}

\author{
Sadika Akhter, Md. Ismail Hossain \\ Department of Pharmacy, University of Asia Pacific, Dhaka, Bangladesh
}

\begin{abstract}
In this study, a solubility enhancing technique, Self-emulsifying drug delivery system (SEDDS), was considered to be developed for Ibuprofen, a poorly soluble drug. Capmul PG 8 was used as a co-solvent. As surfactant, hydrophilic surfactant Cremophor EL was considered. A fixed amount of Ibuprofen was added with fixed amount of excipients. Capmul PG8 showed a good solubilizing capacity which dissolved $300 \mathrm{mg} / \mathrm{ml}$ of Ibuprofen. Cremophor EL also showed a good solubilizing capacity which dissolved $300 \mathrm{mg} / \mathrm{ml}$ of Ibuprofen. Ibuprofen is a poorly soluble drug which was used as experimental drug and $\mathrm{pH} 7.2$ phosphate buffer was used as dissolution medium. The amount of drug was measured form the absorbance of UV spectrophotometer at $221 \mathrm{~nm}$. A 3-level factorial design was carried out to optimize the formulation using design expert software trial version 8.0.3.1. Capmul PG8 and Cremophor EL were used as independent variables where percent drug release at 5, 15 and 45 minutes. The optimized formula contains $24.10 \mathrm{mg}$ Capmul PG8 and $71.02 \mathrm{mg}$ Cremophor EL which releases $27.78 \%$, 44.6\% and 74.24\% ibuprofen at the mentioned time interval. The present study shows that the Capmul PG8 and Cremophor EL have effect the release profile of capsule Ibuprofen. It is found that it is possible to increase the release of Ibuprofen by using Capmul PG8 and Cremophor EL.
\end{abstract}

Key Words: Self-emulsifying drug delivery system (SEDDS), Ibuprofen, Capmul PG 8, Cremophor EL.

\section{INTRODUCTION}

A major problem in drug discovery is that water solubility of $40 \%$ of the new drug molecule is very low resulting inadequate bioavailability, high intrasubject/intersubject changeability and dose proportionality deficiency coupled with elevated hydrophobicity which obstructs the oral delivery of several drugs (Lipinski, 2002; Palmer, 2003). Consequently, it is essential to construct satisfactory formulations which are truly crucial to enhance the solubility and bioavailability of such drugs (Debnath et al., 2011). Self-emulsifying drug delivery systems is one of the most recognized and economically feasible formulation concepts for solving these measures. In advancing the oral bioavailability of poorly water-soluble and lipophilic drugs, SEDDS have been adduced to be sensibly outstanding

\footnotetext{
*Corresponding Author:

Md. Ismail Hossain

Department of Pharmacy

University of Asia Pacific

Dhaka, Bangladesh

E-mail: suhaghossainuap@gmail.com
}

(Gursoy and Benita, 2004). It is well diagnosed that lipid-based formulations can elevate oral bioavailability of insufficiently water-soluble drugs (Pouton, 2000).

SEDDS are isotropic mixture of oil(s), surfactant(s), co-surfactant(s), co-solvent(s) and drug. They form fine oil-in- water emulsions when introduced into aqueous media under gentle agitation (Constantinides, 1995). The aptitude of SEDDS for enhancing the bioavailability of poorly soluble drugs has been assured for at least a decade (Charman et al., 1992). Notwithstanding, major drawbacks of SE formulations are elevated production costs, poor solubility and portability, inadequate drug loading and fewer options of dosage forms as SE formulations are typically formulated as liquids (Tang et al., 2008 ).

There are plenty of arrangements of lipid formulations such as emulsions, surfactant dispersions, oils, solid lipid nanoparticles, liposomes and most importantly SEDDS as it is associated with lipidbased formulations. SEDDS includes the complex 


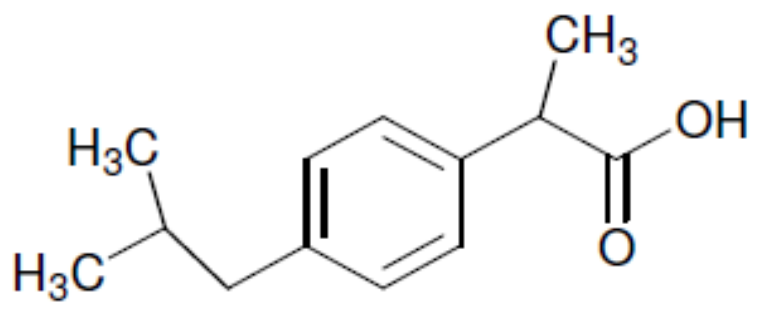

Figure 1: Structure of Ibuprofen.

blends of surfactant and/or co-surfactant, oil/lipid and isotropic combination of drugs as a result emulsion/lipid droplets of close to 100nm (SEDDS) to less than $50 \mathrm{~nm}$ for self-micro emulsifying drug delivery systems (SMEDDS), on dilution with physiological fluid are constituted (Tang et al.,2008). Accordingly, the absorption rate of hydrophobic drugs from the crystalline state becomes limited due to the drug persists in solution form in the gastrointestinal gut escaping the dissolution step (Hauss, 2007). The lipid based formulation strategy has magnetized vast appeal toward amplifying drug solubilisation in the GI tract and to advance the oral bioavailability of BCS Class II and IV drugs (Baboota et al., 2007; Amidon et al., 1995).

The mechanism of action of Self-emulsification is still unsettled. Nevertheless, as stated in Reiss (Reiss, 1975), as soon as the entropy change that accommodates dispersion is outstanding than the energy desired to step up the surface area of the dispersion, self-emulsification takes place. Besides, to introduce a surface between the two phases the energy needed is functionally coupled with the free energy of a classical emulsion formation and these can be characterized by the equation-

$\Delta \mathrm{G}=\sum_{\mathrm{i}} \mathrm{N}_{\mathrm{i}} \pi \mathrm{r}_{\mathrm{i}}^{2} \sigma$

Where $\Delta G$ stands for free energy connected with the process, $\mathrm{N}$ represents the number of droplets of radius, $r$, and $\boldsymbol{\sigma}$ indicates the interfacial energy.

Non-steroidal anti-inflammatory drug (NSAID), Ibuprofen has been extensively administered for mild to moderate pain. A primary requirement for rapid onset of action of Ibuprofen is its express absorption as serum concentrations and analgesic effect of Ibuprofen are interlinked. Nevertheless, following oral administration bioavailability of
Table 1: System used in this experiment.

\begin{tabular}{ll}
\hline Components & Formulation system \\
\hline Oil Phase & Capmul PG8 \\
Surfactants & Cremophor EL \\
\hline
\end{tabular}

Ibuprofen is comparatively poor by reason of insolubility in water (Ghorab and Adeyeye, 1994; Glowka, 2000).

\section{MATERIALS AND METHODS}

\section{Materials}

Pure drug sample of ibuprofen was procured from Xamim, China. Capmul PG8 was procured from Abitec Corporation, Germany and Cremophor EL was procured from BASF, Germany. All other ingredients were obtained commercially and used as received.

\section{Preparation of Calibration Curve of Ibuprofen}

$20 \mathrm{mg}$ of Ibuprofen was taken in a $1000 \mathrm{ml}$ volumetric flask and diluted with phosphate buffer ( $\mathrm{pH}$ 7.2) up to $1000 \mathrm{ml}$. This solution was labeled as stock solution. From the stock solution, $1 \mathrm{ml}, 2 \mathrm{ml}, 3 \mathrm{ml}$, $4 \mathrm{ml}, 5 \mathrm{ml}, 6 \mathrm{ml}, 7 \mathrm{ml}, 8 \mathrm{ml}, 9 \mathrm{ml}, 10 \mathrm{ml}$ was withdrawn with volumetric pipettes, each time the withdrawn solution was taken in different volumetric flask $(10 \mathrm{ml})$ and the volume of each of the solution was made up to $10 \mathrm{ml}$ with phosphate buffer. The absorbances of the solutions were measured by a UV-VIS spectrophotometer of Shimadzu at $221 \mathrm{~nm}$. Using the method, a linear relation $(y=0.0419 x+0.0308)$ was obtained between Ibuprofen concentration $(\mu \mathrm{g} / \mathrm{ml})$ and absorbance values. Correlation Co-efficient $\left(\mathrm{R}^{2}\right)$ value of the calibration curve was found 0.9997 .

\section{Solubility analysis of Ibuprofen in various excipients}

Solubility of Ibuprofen in various components (Capmul PG8 and Cremophor EL) was studied. 1ml of each of the vehicles was added to each captube containing Ibuprofen (300mg). After sealing, mixing of the systems were performed using sonicator and vortex mixer accordingly. Then the mixtures were heated at $\leq 40^{\circ} \mathrm{C}$ in a water bath to facilitate the solubilization and to create clear transparent solution. The mixtures were then kept at room 
Table 2: Formulations of Ibuprofen.

\begin{tabular}{lccccccccc}
\hline $\begin{array}{c}\text { Chemicals } \\
\text { (mg) }\end{array}$ & F1 & F2 & F3 & F4 & F5 & F6 & F7 & F8 & F9 \\
\hline Ibuprofen & 100 & 100 & 100 & 100 & 100 & 100 & 100 & 100 & 100 \\
Capmul PG8 & 100 & 100 & 100 & 300 & 300 & 300 & 500 & 500 & 500 \\
Cremophor EL & 900 & 500 & 700 & 900 & 500 & 700 & 900 & 500 & 700 \\
\hline
\end{tabular}

temperature for 48 hours. After 48 hours, observed the clear solutions were still maintained.

\section{Preparation of SEDDS formulation of Ibuprofen}

SEDDS formulations were developed using Capmul PG8 as oil phase and Cremophor EL as surfactant. Then a series of SEDDS formulation of Ibuprofen were prepared using formulation system (Table 2). Accurately weighed Ibuprofen was placed in a glass vial and Capmul PG8, Cremophor EL were added. Then the components were mixed by gentle vortexing and were heated at $\leq 40^{\circ} \mathrm{C}$ until Ibuprofen was properly dissolved. The SEDDS formulas in the glass vials were then kept at room temperature until further use. $10 \mathrm{mg}$ equivalent weights of Ibuprofen were taken in each 2 size capsule shell and capsules were sealed.

\section{In-vitro Dissolution Study of Ibuprofen SEDDS}

The dissolution studies were carried out using a "USP Dissolution Apparatus 2 (Paddle type)" Phosphate buffer ( $\mathrm{pH}$ 7.2) was used as dissolution media, rpm of baskets paddles were $50 \mathrm{rpm}$ and temperature of dissolution media was maintained at $37 \pm 0.5^{\circ} \mathrm{C}$. One capsule shell was placed into each of one basket with a sinker; these were inserted in the vessels. Dissolution samples were withdrawn at predetermined time intervals of 5, 10, 15, 20, 25, 30, 45, 60 minute. Each time $10 \mathrm{ml}$ of the dissolution samples were withdrawn with a calibrated disposable syringe and media was replaced with fresh portion of phosphate buffer (pH 7.2). Drug content of the dissolution samples i.e. quantity of drug released, was determined by spectrophotometric analysis using "SHIMADZU spectrophotometer". Maximum absorbance for Ibuprofen was taken at 221nm. Phosphate buffer ( $\mathrm{pH} 7.2$ ) was used as blank solution. Absorbance values obtained from the dissolution studies were converted into percent release of drug from the formulations of SEDDS. This is done by comparing the absorbance values with the standard curve.
Table 3: Brand drugs of two pharmaceutical companies.

\begin{tabular}{cc}
\hline Trade Name & Company \\
\hline I 400 & X \\
F 400 & Y \\
\hline
\end{tabular}

In-vitro Dissolution Study of powder Ibuprofen and two brand drugs of two pharmaceutical companies

The dissolution studies for both powder Ibuprofen and two brand drugs of two pharmaceutical companies (Table 3) were carried out using a "USP Dissolution Apparatus 2 (Paddle type)". Phosphate buffer ( $\mathrm{pH}$ 7.2) was used as dissolution media, rpm of baskets paddles were $50 \mathrm{rpm}$ and temperature of dissolution media was maintained at $37 \pm 0.5^{\circ} \mathrm{C}$. One capsule shell was placed into each of one basket; these were inserted in the vessels. Dissolution samples were withdrawn at predetermined time intervals of 5, 10, 15, 20, 25, 30, 45, 60 minute. Each time $10 \mathrm{ml}$ of the dissolution samples were withdrawn with a calibrated disposable syringe and media was replaced with fresh portion of phosphate buffer ( $\mathrm{pH}$ 7.2). Drug content of the dissolution samples i.e. quantity of drug released, was determined by spectrophotometric analysis using "SHIMADZU spectrophotometer". Maximum absorbance for Ibuprofen was taken at $221 \mathrm{~nm}$. Phosphate buffer ( $\mathrm{pH}$ 7.2) was used as blank solution. Absorbance values obtained from the dissolution studies were converted into percent release. This is done by comparing the absorbance values with the standard curve.

\section{Response surface methodology}

Many statistical experimental designs have been recognized as useful technique to optimize the process variables. For this purpose, a computer based optimization technique with response surface methodology (RSM) using a polynomial equation has been widely used. Different types of RSM design include 3-level factorial design, Box-Behnken design and D-optimal design. Based on the principal of design of experiments (DoE), the methodology encompasses the use of various types of experimental designs, generation of polynomial equations, and mapping of the response over the experimental domain to determine the optimum formulation. Response Surface Methodology (RSM) is used only when a few significant factors are involved in 
Table 4: Formulations (F1, F2 and F3) which had fixed amount of Capmul PG8 but variable amount of Cremophor EL.

\begin{tabular}{llll}
\hline Chemicals (mg) & F1 & F2 & F3 \\
\hline Ibuprofen & 100 & 100 & 100 \\
Capmul PG8 & 100 & 100 & 100 \\
Cremophor EL & 900 & 500 & 700 \\
\hline
\end{tabular}

optimization. The technique requires minimum experimentation and time, thus providing to be far more effective and cost-effective than the conventional methods of formulating sustained release dosage forms.

A 3-level factorial design was employed as per the standard protocol. The amounts of Capmul PG8 ( $\left.\mathrm{X}_{1}\right)$ and Cremophor EL $\left(\mathrm{X}_{2}\right)$ were selected as dependant variables, studied at 3 levels each. The central point $(0,0)$ was studied in quintuplicate. All other formulation and processing variables were kept invariant throughout the study. Total 13 experimental runs were conduced. Responses considered are $\mathrm{Y}_{1}$-Drug releases at 5 minute, $Y_{2}$ - Drug release at 15 minute, $\mathrm{Y}_{3}$ - Drug release at 45 minute were taken as the response variables.

\section{Optimization of Data Analysis}

Various RSM computations for the current optimization study were performed employing Design Expert software (Design Expert 8.0.3.1 Trial Version, Stat-Ease Inc, Minneapolis, MN). Polynomial models including interaction and quadratic terms were generated for all the response variables using multiple linear regression analysis (MLRA) approach. The general form of the MLRA model is represented as equation:

$\left(\mathrm{Y}=\mathrm{A}_{0}+\mathrm{A}_{1} \mathrm{X}_{1}+\mathrm{A}_{2} \mathrm{X}_{2}+\mathrm{A}_{3} \mathrm{X}_{1} \mathrm{X}_{2}+\mathrm{A}_{4} \mathrm{X}_{1}^{2}+\mathrm{A}_{5} \mathrm{X}_{2}^{2}\right)$

Where, $A_{0}$ is the intercept representing the arithmetic average of all quantitative outcomes of 13 runs; $A_{1}$ to $A_{5}$ are the coefficients computed from the observed experimental values of $\mathrm{Y}$; and $\mathrm{X}_{1}$ and $\mathrm{X}_{2}$ are the coded levels of the independent variable(s). The terms $X_{1} X_{2}$ and $X_{1}(i=1$ to 2$)$ represent the interaction and quadratic terms, respectively. Statistical validity of the polynomials was established on the basis of ANOVA provision in the Design Expert software. Subsequently, the feasibility and grid searches were performed to locate the composition of optimum formulations. Also, the 3-D
Table 5: Formulations (F4, F5 and F6) which had fixed amount of Capmul PG8 but variable amount of Cremophor EL.

\begin{tabular}{llll}
\hline Chemicals (mg) & F4 & F5 & F6 \\
\hline Ibuprofen & 100 & 100 & 100 \\
Capmul PG8 & 300 & 300 & 300 \\
Cremophor EL & 900 & 500 & 700 \\
\hline
\end{tabular}

response surface graphs and 2-D contour plots were constructed in MS-Excel environment using the output files generated by the Design Expert software.

\section{RESULTS AND DISCUSSION}

Ibuprofen is a poorly water soluble drug. It was not seemed to be soluble in normal condition, but it was soluble when heated in a steam bath for few minutes. The solubility and the dissolution of Ibuprofen were very slow. But it was made soluble and brought into the dissolution first by the addition of co-solvent (Capmul PG8) and surfactant (Cremophor EL). Drug release kinetics was done by basket method using $\mathrm{pH} 7.2$ phosphate buffer as dissolution medium at room temperature $37 \pm 0.5^{\circ} \mathrm{C}$ at $50 \mathrm{rpm}$ speed. The sample was collected for 1 hour studies and percentage of drug release at different time interval was calculated from the UV absorbance reading. $10 \mathrm{ml}$ syringe was used to take $10 \mathrm{ml}$ sample from each sample basket and $10 \mathrm{ml}$ of fresh phosphate buffer was added after the sample was taken into each sample basket. Sample was filtered and percent (\%) release of Ibuprofen was calculated from UV absorbance reading of sample.

\section{Percent (\%) release for Ibuprofen SEDDS formula- tion}

The formulation (Table 2) F1, F2, F3, F4, F5, F6, F7, F8 and F9 showed about $25.90658 \%, 24.55585 \%$, $31.01415 \%$ ， $44.07044 \% ， \quad 78.64965 \% ， \quad 94.36742 \%$, $25.33913 \%, 51.42469 \%$ and $42.0337 \%$ release respectively within 1 hour.

Dissolution rate of Ibuprofen was increased by the use of co-solvent and surfactant which is due to the wettabilitty and spreadability of the precipitated drug by reducing aggregations in the readily soluble state. 


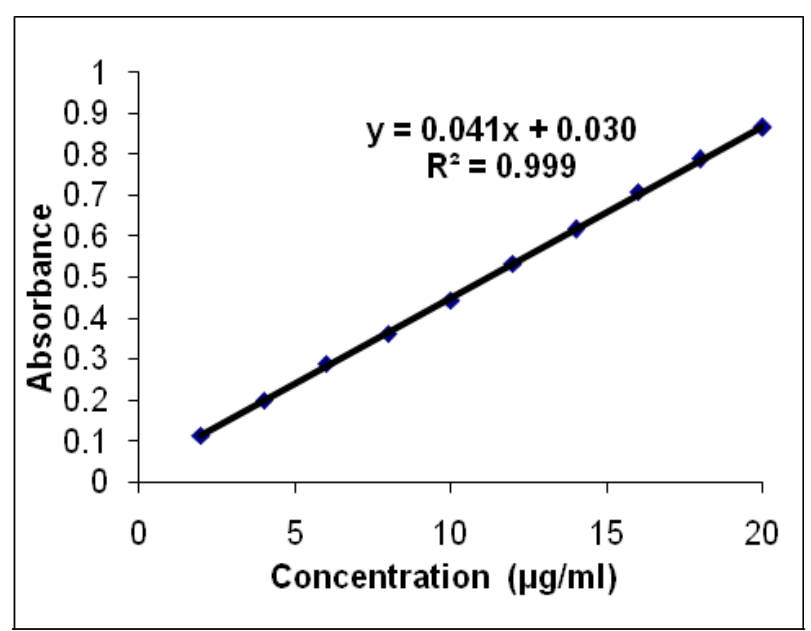

Figure 2: Calibration curve of Ibuprofen.

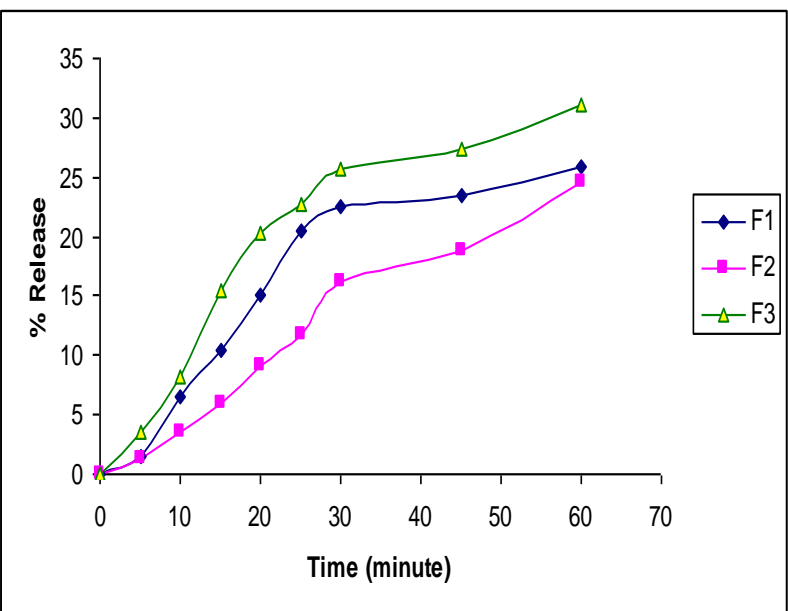

Figure 4: Percent (\%) release of Ibuprofen from different relations containing fixed amount of Capmul PG8 (10mg) and variable amount of Cremophor EL.

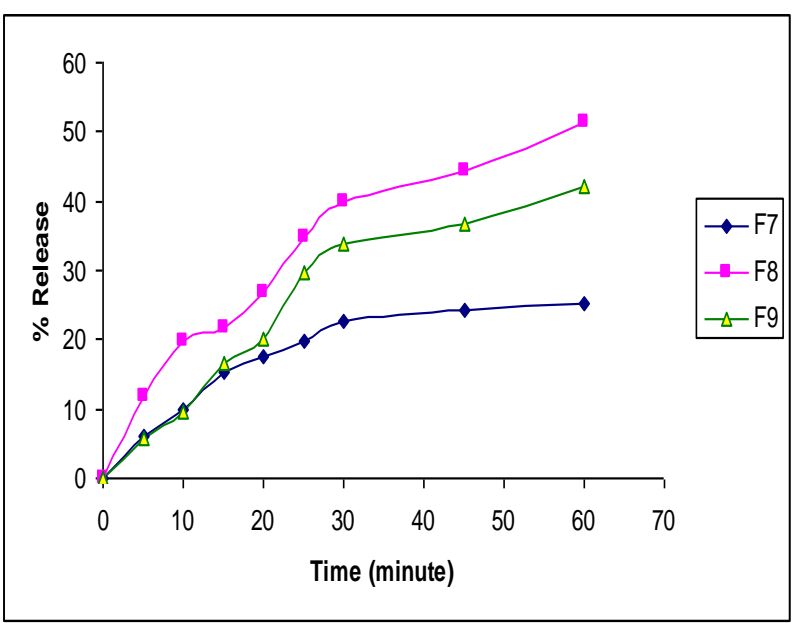

Figure 6: Percent (\%) release of Ibuprofen from different relations containing fixed amount of Capmul PG8 (50mg) and variable amount of Cremophor EL.

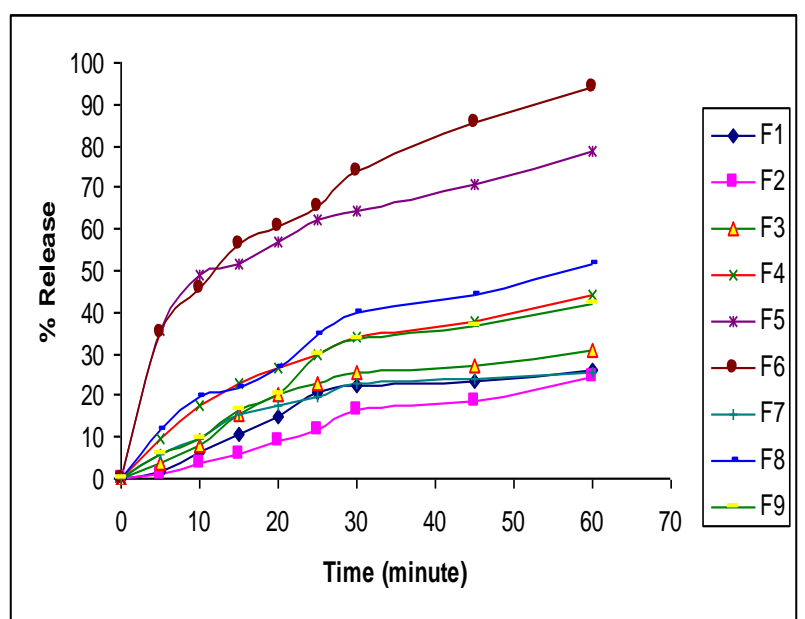

Figure 3: Percent drug release of Ibuprofen at different time intervals

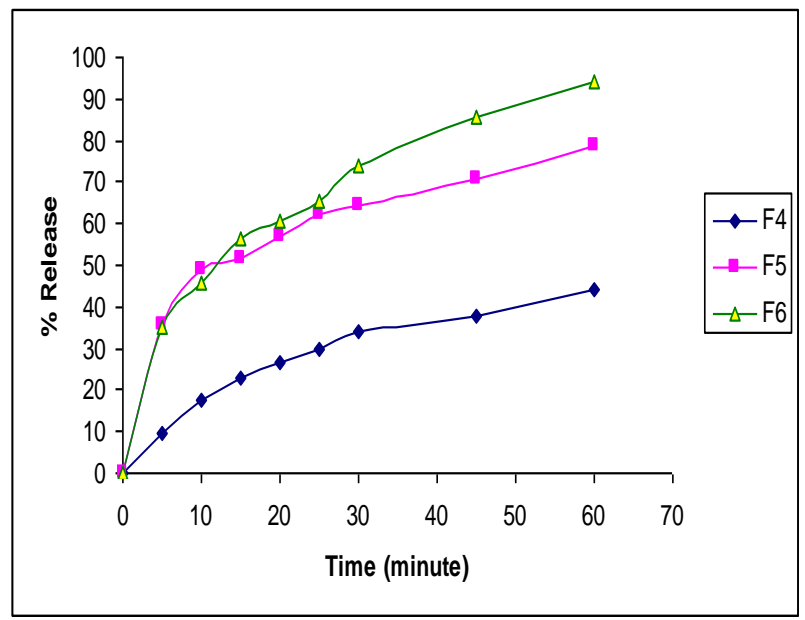

Figure 5: Percent (\%) release of Ibuprofen from different relations containing fixed amount of Capmul PG8 $(30 \mathrm{mg})$ and variable amount of Cremophor EL.

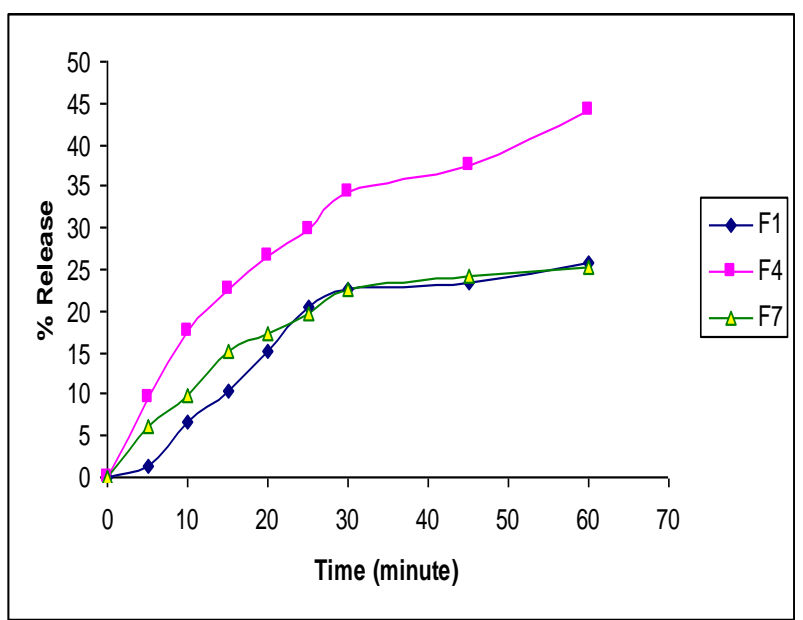

Figure 7: Percent (\%) release of Ibuprofen from different relations containing fixed amount of Cremophor EL (90mg) and variable amount of Capmul PG8. 


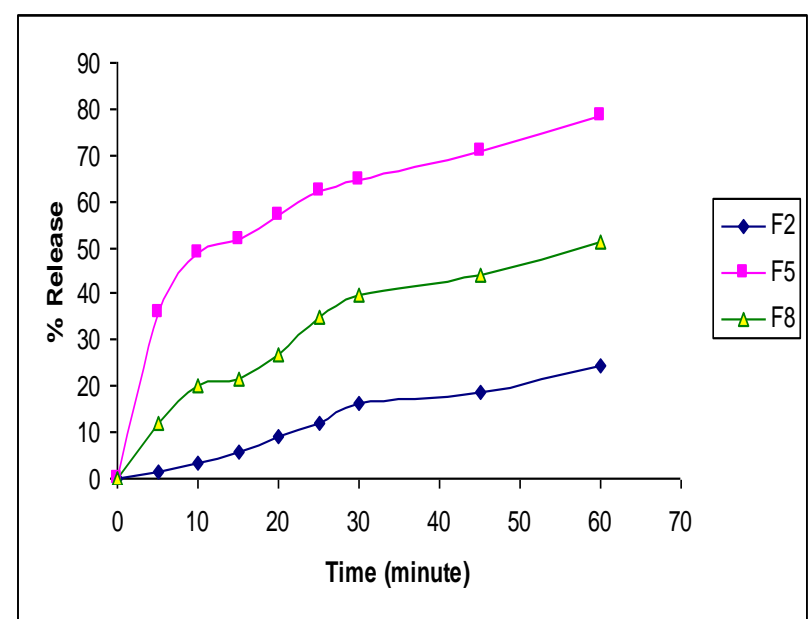

Figure 8: Percent (\%) release of Ibuprofen from different relations containing fixed amount of Cremophor EL (50mg) and variable amount of Capmul PG8.

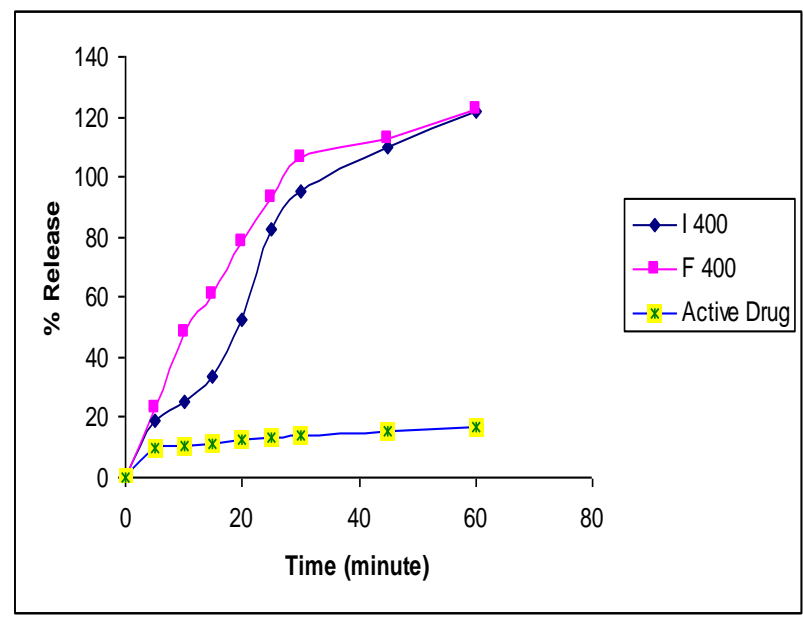

Figure 10: Percent (\%) release of powder Ibuprofen and two brand drugs of two pharmaceutical companies.

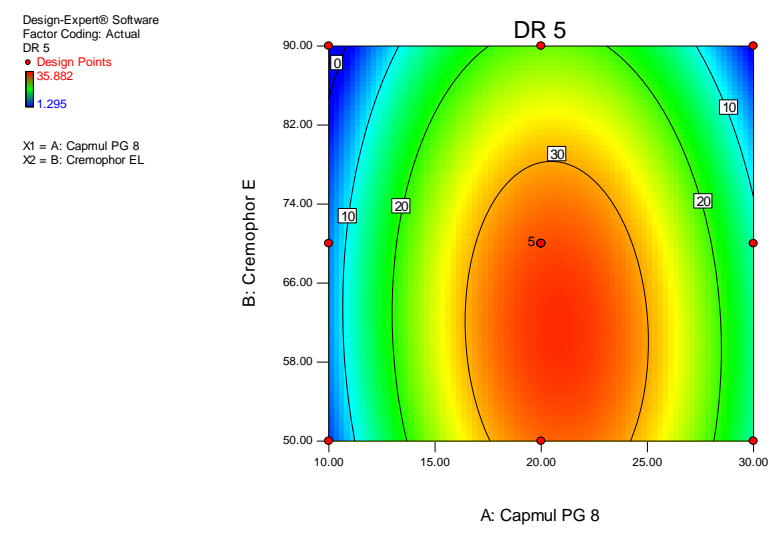

Figure 12: Model graph (contour) for the response DR5 for Capmul PG8 and Cremophor EL based Ibuprofen SEDDS formulation.

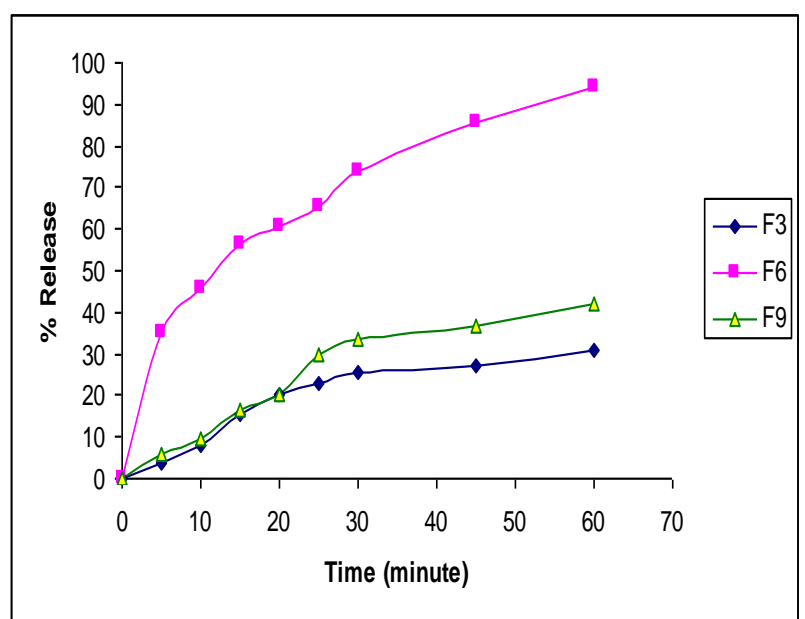

Figure 9: Percent (\%) release of Ibuprofen from different relations containing fixed amount of Cremophor EL (70mg) and variable amount of Capmul PG8.

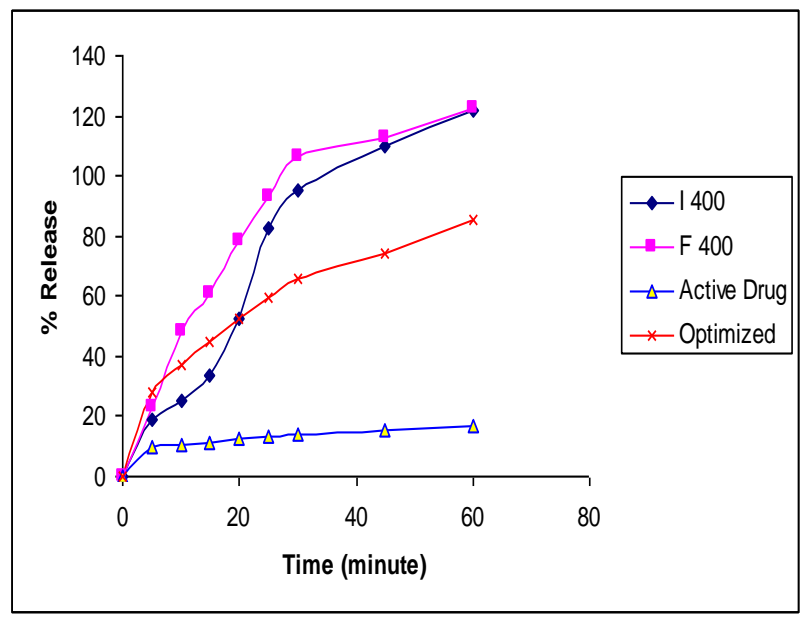

Figure 11: Percent (\%) release of powder Ibuprofen, two brand drugs of two pharmaceutical companies and statistically selected optimized formulation.

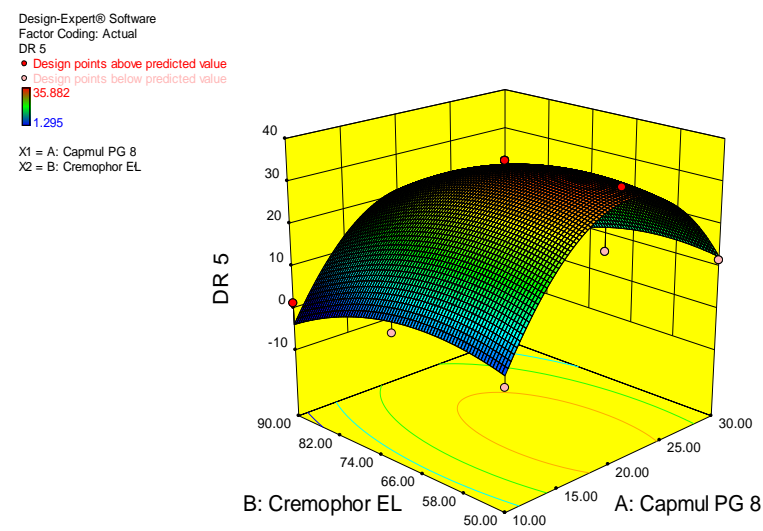

Figure 13: Model graph (3 D surface) for the response DR5 for Capmul PG8 and Cremophor EL based Ibuprofen SEDDS formulation. 


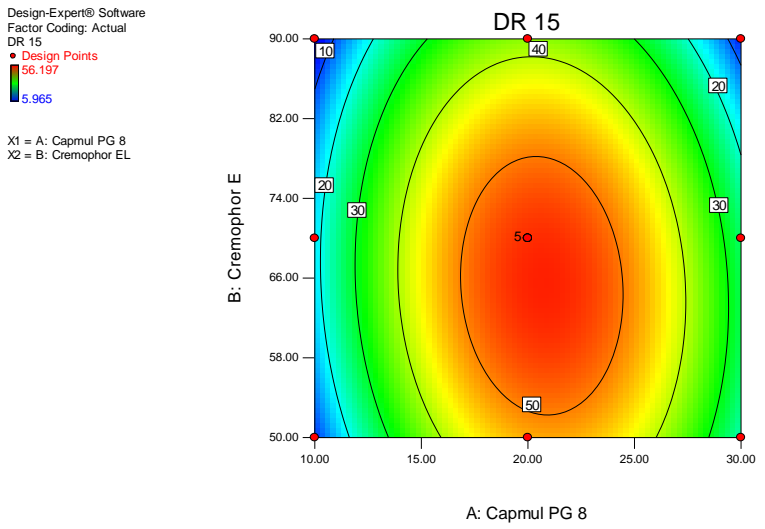

Figure 14: Model graph (contour) for the response DR15 for Capmul PG8 and Cremophor EL based Ibuprofen SEDDS formulation.

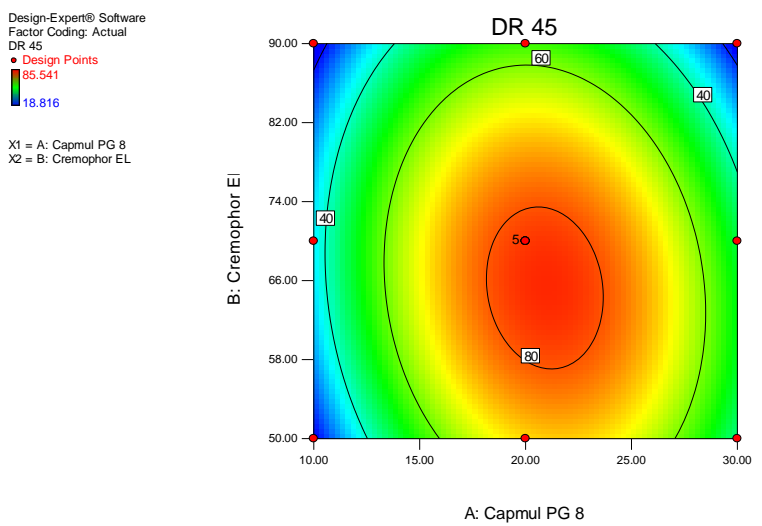

Figure 16: Model graph (contour) for the response DR45 for Capmul PG8 and Cremophor EL based Ibuprofen SEDDS formulation.

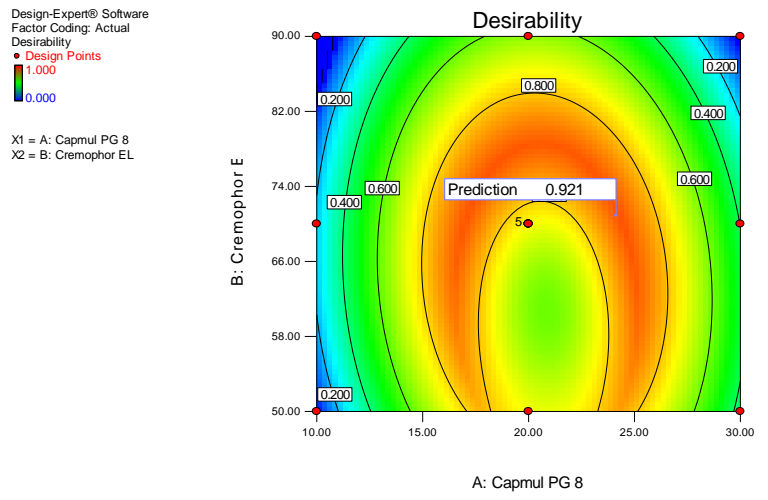

Figure 18: Desiarbility graph (contour) for Capmul PG8 and Cremophor EL based Ibuprofen SEDDS formulation.

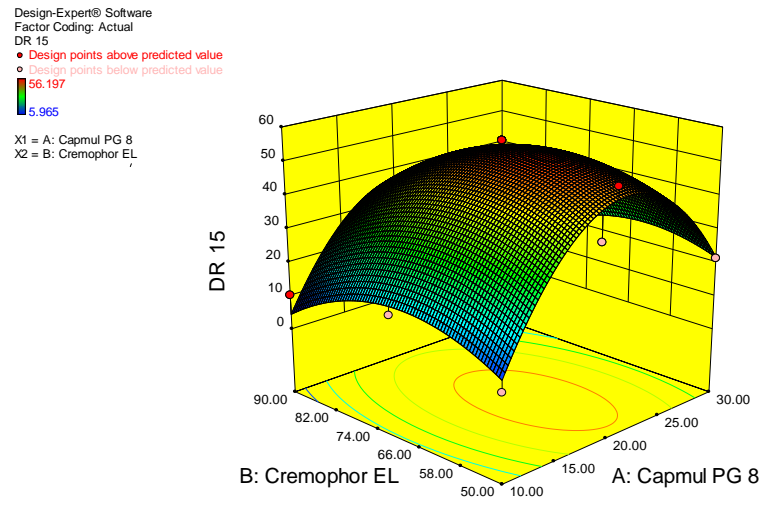

Figure 15: Model graph (3 D surface) for the response DR15 for Capmul PG8 and Cremophor EL based Ibuprofen SEDDS formulation.

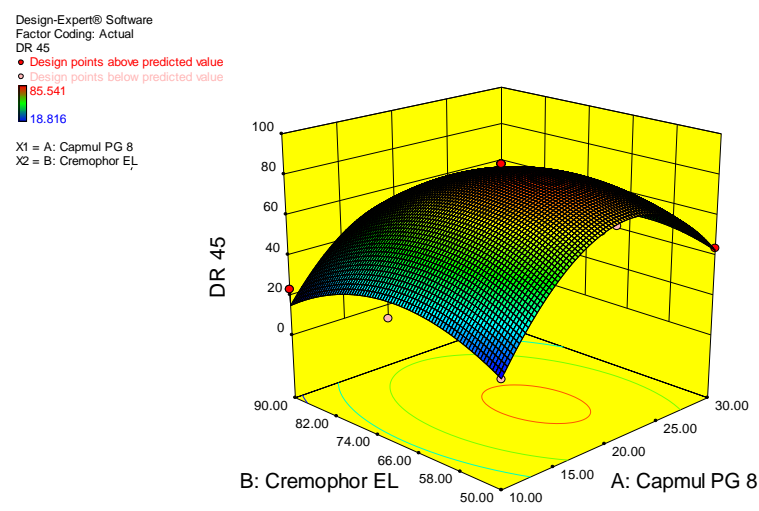

Figure 17: Model graph (3 D surface) for the response DR45 for Capmul PG8 and Cremophor EL based Ibuprofen SEDDS formulation.

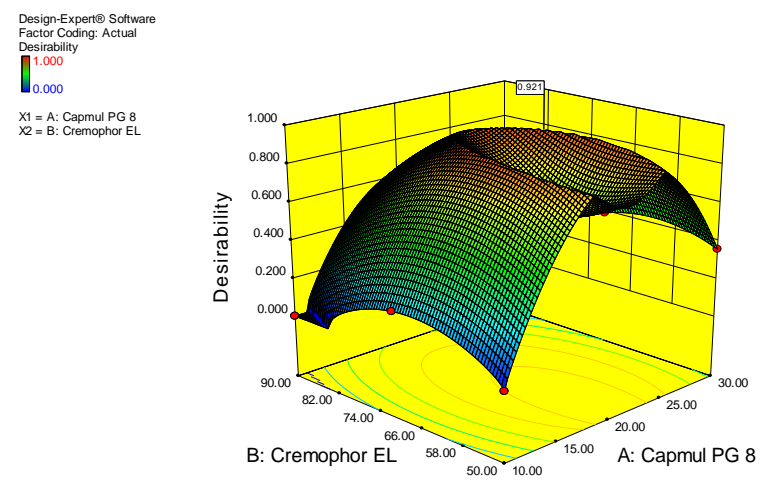

Figure 19: Desiarbility graph (3 D Surface) for Capmul PG8 and Cremophor EL based Ibuprofen SEDDS formulation. 
Table 6: Formulations (F7, F8 and F9) which had fixed amount of Capmul PG8 but variable amount of Cremophor EL.

\begin{tabular}{llll}
\hline Chemicals (mg) & F7 & F8 & F9 \\
\hline Ibuprofen & 100 & 100 & 100 \\
Capmul PG8 & 500 & 500 & 500 \\
Cremophor EL & 900 & 500 & 700 \\
\hline
\end{tabular}

So, it can be considered that the vehicles acted here as emulsifying agents for the liberated drug, thus preventing the formulation of any water insoluble surface layers. Although the liberated drug remained un-dissolved in the dissolution medium when its concentration exceeded its saturation solubility, it was emulsified in a finely divided state because of surface activity of the dissolved vehicle.

Percent (\%) release for the formulations which had the fixed amount of Capmul PG8 or Cremophor EL Where the amount of Capmul PG8 was fixed but the amount of Cremophor EL was different

In case of formulation F1, F2, F3 (Table 4) the amount of Capmul PG8 was 10mg but the amount of Cremophor EL were $90 \mathrm{mg}, 50 \mathrm{mg}$, and $70 \mathrm{mg}$ respectively. The combination of drug (10mg), Capmul PG8 (10mg) and Cremophor EL (70mg) showed highest \% release that was $31.014 \%$ (Figure 4).

In case of formulation F4, F5, F6 (Table 5) the amount of Capmul PG8 was 30mg but the amount of Cremophor EL were $90 \mathrm{mg}$, 50mg, and $70 \mathrm{mg}$ respectively. The combination of drug $(10 \mathrm{mg})$, Capmul PG8 (30mg) and Cremophor EL (70mg) showed highest \% release that was $94.367 \%$ (Figure 5).

In case of formulation F7, F8, F9 (Table 6) the amount of Capmul PG8 was 50mg but the amount of Cremophor EL were $90 \mathrm{mg}, 50 \mathrm{mg}$, and $70 \mathrm{mg}$ respectively. The combination of drug (10mg), Capmul PG8 (50mg) and Cremophor EL (50mg) showed highest \% release that was $51.425 \%$ (Figure 6).

Table 8: Formulations (F2, F5 and F8) which had fixed amount of Cremophor EL but variable amount of Capmul PG8.

\begin{tabular}{llll}
\hline Chemicals (mg) & F2 & F5 & F8 \\
\hline Ibuprofen & 100 & 100 & 100 \\
Capmul PG8 & 100 & 300 & 500 \\
Cremophor EL & 500 & 500 & 500 \\
\hline
\end{tabular}

Table 7: Formulations (F1, F4 and F7) which have fixed amount of Cremophor EL but variable amount of Capmul PG8.

\begin{tabular}{llll}
\hline Chemicals (mg) & F1 & F4 & F7 \\
\hline Ibuprofen & 100 & 100 & 100 \\
Capmul PG8 & 100 & 300 & 500 \\
Cremophor EL & 900 & 900 & 900 \\
\hline
\end{tabular}

Here we can predict that, co-relation was presented between formulations F3 and F6 but there was no co-relation found formulation F8 with F3 and F6 because there was no sufficient amount of Cremophor EL to react with Capmul PG8 and give sufficient emulsifying effect.

Where the amount of Cremophor EL is fixed but the amount of Capmul PG8 is different

In case of formulation F1, F4, F7 (Table 7) the amount of Cremophor EL was $90 \mathrm{mg}$ but the amount of Capmul PG8 were $10 \mathrm{mg}$, 30mg, and $50 \mathrm{mg}$ respectively. The combination of drug (10mg), Cremophor EL (90mg) and Capmul PG8 (30mg) showed highest \% release that was $44.07 \%$ (Figure 7 ).

In case of formulation F2,F5, F8 (Table 8) the amount of Cremophor EL was 50mg but the amount of Capmul PG8 were $10 \mathrm{mg}, 30 \mathrm{mg}, 50 \mathrm{mg}$. The combination of drug $(10 \mathrm{mg})$, Cremophor EL $(50 \mathrm{mg})$ and Capmul PG8 (30mg) showed height \% release that was $78.65 \%$ (Figure 8 ).

In case of formulation F3,F6, F9 (Table 9) the amount of Cremophor EL was 70mg but the amount of Capmul PG8 were $10 \mathrm{mg}, 30 \mathrm{mg}, 50 \mathrm{mg}$. The combination of drug (10mg), Cremophor EL (70mg) and Capmul PG8 (30mg) showed height \% release that was $94.367 \%$ (Figure 9).

Here we can predict that, formulations F4, F5 and F6 showed highest \% release when the amount Capmul PG8 was $30 \mathrm{mg}$. In case of SEDDS if the emulsifying

Table 9: Formulations (F3, F6 and F9) which had fixed amount of Cremophor EL but different amount of Capmul PG8.

\begin{tabular}{llll}
\hline Chemicals (mg) & F3 & F6 & F9 \\
\hline Ibuprofen & 100 & 100 & 100 \\
Capmul PG8 & 100 & 300 & 500 \\
Cremophor EL & 700 & 700 & 700 \\
\hline
\end{tabular}


Table 10: Percent (\%) release of powder Ibuprofen and two brand drugs of two pharmaceutical companies.

\begin{tabular}{clll}
\hline Time (minute) & I 400 & F 400 & Active Drug \\
\hline 0 & 0 & 0 & 0 \\
5 & 18.558 & 22.854 & 9.987 \\
10 & 24.916 & 48.377 & 10.634 \\
15 & 33.255 & 60.773 & 11.301 \\
20 & 52.334 & 78.276 & 12.299 \\
25 & 82.415 & 92.773 & 13.269 \\
30 & 95.312 & 106.362 & 14.133 \\
45 & 109.642 & 112.410 & 15.345 \\
60 & 122.062 & 122.467 & 16.968 \\
\hline
\end{tabular}

time and other parameters are justified Capmul PG8 (30 mg) which is a co-solvent increases the emulsifying capacity of Cremophor EL.

Percent (\%) release of powder Ibuprofen and two brand drugs of two different pharmaceutical companies

For both brand drugs' percent release were $122.062 \%$ of I 400 and $122.469 \%$ of F 400 . Percent release of powder active drug was $16.969 \%$. Here the presence of excipients in brand drugs increased the percent release of Ibuprofen.

Percent (\%) release of powder Ibuprofen, two brand drugs of two different pharmaceutical companies and statistically selected optimized formulation.

For both brand drugs percent release were $122.062 \%$ of I 400 and $122.469 \%$ of F 400 . Percent release of

Table 11: Percent ( $\%)$ release of powder Ibuprofen, two brand drugs of two pharmaceutical companies and statistically selected optimized formulation.

\begin{tabular}{|c|c|c|c|c|}
\hline 苟 & $\underset{8}{\stackrel{4}{ }}$ & 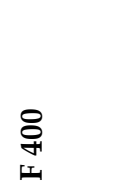 & 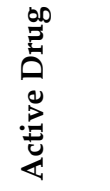 & 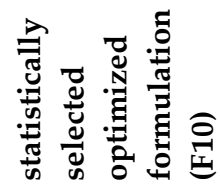 \\
\hline 0 & 0 & 0 & 0 & 0 \\
\hline 5 & 18.558 & 22.854 & 9.987 & 27.784 \\
\hline 10 & 24.916 & 48.377 & 10.634 & 36.888 \\
\hline 15 & 33.255 & 60.773 & 11.301 & 44.602 \\
\hline 20 & 52.334 & 78.276 & 12.299 & 52.809 \\
\hline 25 & 82.415 & 92.773 & 13.269 & 59.789 \\
\hline 30 & 95.312 & 106.362 & 14.133 & 65.758 \\
\hline 45 & 109.642 & 112.410 & 15.345 & 74.247 \\
\hline 60 & 122.062 & 122.467 & 16.968 & 85.425 \\
\hline
\end{tabular}

powder active drug was $16.969 \%$ and percent release of statistically selected optimized formulation was $85.424 \%$. Here the \% release of statistically selected optimized formulation was quite closer to the $\%$ release of brand drugs.

\section{ANOVA for Response Surface Quadratic Model Response 1 at DR5}

The Model F-value of 11.88 implies the model is significant. There is only a $0.26 \%$ chance that a "Model F-Value" this large could occur due to noise. Values of "Prob > F" less than 0.0500 indicate model terms are significant. In this case $\mathrm{A}^{2}$ are significant model terms. Values greater than 0.1000 indicate the model terms are not significant. If there are many insignificant model terms (not counting those required to support hierarchy), model reduction may improve the model.

\begin{tabular}{llll}
\hline Std. Dev. & 6.61 & R-Squared & 0.8946 \\
Mean & 19.24 & Adj R-Squared & 0.8193 \\
C.V. \% & 34.36 & Pred R-Squared & 0.0567 \\
PRESS & 2738.16 & Adeq Precision & 8.233 \\
\hline
\end{tabular}

The "Pred R-Squared" of 0.0567 is not as close to the "Adj R-Squared" of 0.8193 as one might normally expect. This may indicate a large block effect or a possible problem with your model and/or data. Things to consider are model reduction, response tranformation, outliers, etc."Adeq Precision" measures the signal to noise ratio. A ratio greater than 4 is desirable. Your ratio of 8.233 indicates an adequate signal. This model can be used to navigate the design space.

\begin{tabular}{|c|c|c|c|c|c|c|}
\hline Factor & $\begin{array}{l}\text { Co- } \\
\text { efficient } \\
\text { Estimate }\end{array}$ & df & $\begin{array}{l}\text { Stan- } \\
\text { dard } \\
\text { Error }\end{array}$ & $\begin{array}{l}95 \% \\
\text { CI } \\
\text { Low }\end{array}$ & $\begin{array}{l}95 \% \\
\text { CI } \\
\text { High }\end{array}$ & VIF \\
\hline Intercept & 33.20 & 1 & 2.75 & 26.71 & 39.69 & \\
\hline A-Capmul PG 8 & 2.89 & 1 & 2.70 & -3.49 & 9.28 & 1.00 \\
\hline B-Cremophor EL & -5.30 & 1 & 2.70 & -11.68 & 1.08 & 1.00 \\
\hline $\mathrm{AB}$ & -1.45 & 1 & 3.31 & -9.27 & 6.37 & 1.00 \\
\hline$A^{2}$ & -24.19 & 1 & 3.98 & -33.60 & -14.78 & 1.17 \\
\hline $\mathrm{B}^{2}$ & -6.05 & 1 & 3.98 & -15.46 & 3.36 & 1.17 \\
\hline
\end{tabular}

\section{Response 2 at DR 15}

The Model F-value of 14.61 implies the model is significant. There is only a $0.14 \%$ chance that a "Model F-Value" this large could occur due to noise.Values of "Prob > F" less than 0.0500 indicate model terms are significant.In this case $\mathrm{A}^{2}$ are 
Table 12: Analysis of variance.

\begin{tabular}{lllllll}
\hline Source & Sum of Squares & df & Mean Square & F Value & p-value Prob $>$ F \\
\hline Model & 2596.79 & 5 & 519.36 & 11.88 & 0.0026 & significant \\
A-Capmul PG 8 & 50.18 & 1 & 50.18 & 1.15 & 0.3196 \\
B-Cremophor EL & 168.54 & 1 & 168.54 & 3.85 & 0.0904 \\
AB & 8.44 & 1 & 8.44 & 0.19 & 0.6737 \\
$\mathrm{~A}^{2}$ & 1616.41 & 1 & 1616.41 & 36.97 & 0.0005 \\
B $^{2}$ & 101.19 & 1 & 101.19 & 2.31 & 0.1720 \\
Residual & 306.04 & 7 & 43.72 & & \\
Lack of Fit & 306.04 & 3 & 102.01 & & \\
Pure Error & 0.000 & 4 & 0.000 & & \\
Cor Total & 2902.83 & 12 & & & \\
\hline
\end{tabular}

Table 13: Analysis of variance.

\begin{tabular}{lllllll}
\hline Source & Sum of Squares & df & Mean Square & F Value & p-value Prob > F \\
\hline Model & 4926.94 & 5 & 985.39 & 14.61 & 0.0014 & significant \\
A-Capmul PG 8 & 76.56 & 1 & 76.56 & 1.14 & 0.3220 \\
B-Cremophor EL & 159.21 & 1 & 159.21 & 2.36 & 0.1683 \\
AB & 29.21 & 1 & 29.21 & 0.43 & 0.5315 \\
A $^{2}$ & 2881.04 & 1 & 2881.04 & 42.72 & 0.0003 \\
B $^{2}$ & 344.88 & 1 & 344.88 & 5.11 & 0.0582 \\
Residual & 472.12 & 7 & 67.45 & & \\
Lack of Fit & 472.12 & 3 & 157.37 & & \\
Pure Error & 0.000 & 4 & 0.000 & & \\
Cor Total & 5399.06 & 12 & & & \\
\hline
\end{tabular}

Table 14: Analysis of variance.

\begin{tabular}{lllllll}
\hline Source & Sum of Squares & df & Mean Square & F Value & p-value Prob > F \\
\hline Model & 8964.44 & 5 & 1792.89 & 17.30 & 0.0008 & significant \\
A-Capmul PG 8 & 209.14 & 1 & 209.14 & 2.02 & 0.1984 \\
B-Cremophor EL & 396.16 & 1 & 396.16 & 3.82 & 0.0915 \\
AB & 153.41 & 1 & 153.41 & 1.48 & 0.2632 \\
$\mathrm{~A}^{2}$ & 4525.81 & 1 & 4525.81 & 43.66 & 0.0003 \\
$\mathrm{~B}^{2}$ & 927.80 & 1 & 927.80 & 8.95 & 0.0202 \\
Residual & 725.57 & 7 & 103.65 & & \\
Lack of Fit & 725.57 & 3 & 241.86 & & \\
Pure Error & 0.000 & 4 & 0.000 & & \\
Cor Total & 9690.00 & 12 & & & \\
\hline
\end{tabular}

significant model terms.Values greater than 0.1000 indicate the model terms are not significant.If there are many insignificant model terms (not counting those required to support hierarchy), model reduction may improve the model.

\begin{tabular}{llll}
\hline Std. Dev. & 8.21 & R-Squared & 0.9126 \\
Mean & 33.88 & Adj R-Squared & 0.8501 \\
C.V. \% & 24.24 & Pred R-Squared & 0.2241 \\
PRESS & 4189.32 & Adeq Precision & 8.871 \\
\hline
\end{tabular}

The "Pred R-Squared" of 0.2241 is not as close to the "Adj R-Squared" of 0.8501 as one might normally expect. This may indicate a large block effect or a possible problem with your model and/or data. Things to consider are model reduction, response tranformation, outliers, etc. "Adeq Precision" measures the signal to noise ratio. A ratio greater than 4 is desirable. Your ratio of 8.871 indicates an 
adequate signal. This model can be used to navigate the design space.

\begin{tabular}{|c|c|c|c|c|c|c|}
\hline Factor & $\begin{array}{l}\text { Co- } \\
\text { efficient } \\
\text { Estimate }\end{array}$ & df & $\begin{array}{l}\text { Stan- } \\
\text { dard } \\
\text { Error }\end{array}$ & $\begin{array}{l}95 \% \\
\text { CI } \\
\text { Low }\end{array}$ & $\begin{array}{l}95 \% \\
\text { CI } \\
\text { High }\end{array}$ & VIF \\
\hline Intercept & 53.95 & 1 & 3.41 & 45.88 & 62.01 & \\
\hline A-Capmul PG 8 & 3.57 & 1 & 3.35 & -4.36 & 11.50 & 1.00 \\
\hline B-Cremophor EL & -5.15 & 1 & 3.35 & -13.08 & 2.78 & 1.00 \\
\hline $\mathrm{AB}$ & -2.70 & 1 & 4.11 & -12.41 & 7.01 & 1.00 \\
\hline$A^{2}$ & -32.30 & 1 & 4.94 & -43.98 & -20.61 & 1.17 \\
\hline$B^{2}$ & -11.17 & 1 & 4.94 & -22.86 & 0.51 & 1.17 \\
\hline
\end{tabular}

\section{Response 3 at DR 45}

The Model F-value of 17.30 implies the model is significant. There is only a $0.08 \%$ chance that a "Model F-Value" this large could occur due to noise.Values of "Prob > F" less than 0.0500 indicate model terms are significant.In this case $\mathrm{A}^{2}, \mathrm{~B}^{2}$ are significant model terms.Values greater than 0.1000 indicate the model terms are not significant. If there are many insignificant model terms (not counting those required to support hierarchy),model reduction may improve your model.

\begin{tabular}{llll}
\hline Std. Dev. & 10.18 & R-Squared & 0.9251 \\
Mean & 54.67 & Adj R-Squared & 0.8716 \\
C.V. \% & 18.62 & Pred R-Squared & 0.4125 \\
PRESS & 5693.13 & Adeq Precision & 9.719 \\
\hline
\end{tabular}

The "Pred R-Squared" of 0.4125 is not as close to the "Adj R-Squared" of 0.8716 as one might normally expect. This may indicate a large block effect or a possible problem with your model and/or data. Things to consider are model reduction, response tranformation, outliers, etc."Adeq Precision" measures the signal to noise ratio. A ratio greater than 4 is desirable. Your ratio of 9.719 indicates an adequate signal. This model can be used to navigate the design space.

\begin{tabular}{|c|c|c|c|c|c|c|}
\hline Factor & $\begin{array}{l}\text { Co- } \\
\text { efficient } \\
\text { Estimate }\end{array}$ & df & $\begin{array}{l}\text { Stan- } \\
\text { dard } \\
\text { Error }\end{array}$ & $\begin{array}{l}95 \% \\
\text { CI } \\
\text { Low }\end{array}$ & $\begin{array}{l}95 \% \\
\text { CI } \\
\text { High }\end{array}$ & VIF \\
\hline Intercept & 81.81 & 1 & 4.23 & 71.82 & 91.81 & \\
\hline A-Capmul PG 8 & 5.90 & 1 & 4.16 & -3.92 & 15.73 & 1.00 \\
\hline B-Cremophor EL & -8.13 & 1 & 4.16 & -17.95 & 1.70 & 1.00 \\
\hline $\mathrm{AB}$ & -6.19 & 1 & 5.09 & -18.23 & 5.84 & 1.00 \\
\hline$A^{2}$ & -40.48 & 1 & 6.13 & -54.97 & -25.99 & 1.17 \\
\hline$B^{2}$ & -18.33 & 1 & 6.13 & -32.81 & -3.84 & 1.17 \\
\hline
\end{tabular}

\section{Formulation optimization of Ibuprofen}

To optimize Ibuprofen formulation, Capmul PG8 and Cremophor EL were evaluated at different
Table 15: Optimization Criteria for DR5, DR15 and DR45.

\begin{tabular}{llll}
\hline Time (minute) & Lower limit & Upper limit & Target \\
\hline DR5 & 1.295 & 35.882 & 30 \\
DR15 & 5.965 & 56.197 & 55 \\
DR45 & 18.816 & 85.541 & 85 \\
\hline
\end{tabular}

concentration. The lowest DR5 value was obtained 1.295. Similarly lowest DR15 and DR45 values were 5.965 and 18.816 respectively (Table 15). Setting the target for responses empirically like $30 \%$ release for DR5, 55\% release for DR15, and 85\% release for DR45 provided the following solutions among which No. 1 was selected for its higher desirability value. The selected formulation possesses committed the target value for DR5 but slightly lower value obtained for DR15 and DR45 than the target value. That's why instead of statistically selected one formulation F10 was selected for further processing.

From the experimental Ibuprofen formulation it has been seen that after 1 hours of dissolution 85.42469 $\%$ of Ibuprofen was released. The dependant responses (DR5, DR15, and DR45) obtained are $27.78,44.6$ and 74.24 respectively whereas the projected responses were 30,55 and 85 respectively (Table 18). This shows closeness between theoretically optimized and actual response values.

\section{CONCLUSION}

The study was designed to optimize Self-

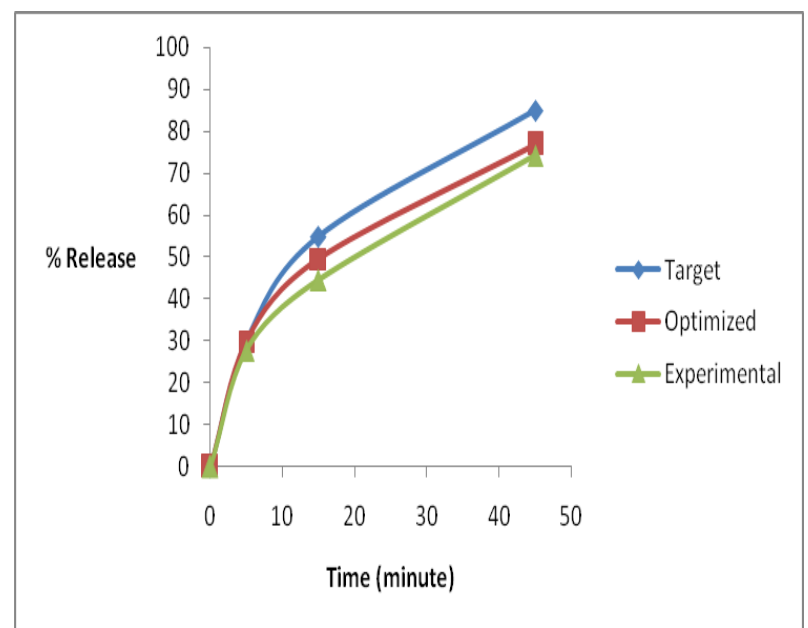

Figure 20: Percent drug release of Ibuprofen from target, optimized and experiment formulation. 
Table 16: Optimized solution obtained from Design expert software for Ibuprofen loaded Capmul PG8 and Cremophor EL based SEDDS formulation optimization.

\begin{tabular}{clllllll}
\hline Number & Capmul PG 8 & Cremophor EL & DR 5 & DR 15 & DR 45 & Desirability \\
\hline 1 & 24.10 & 71.02 & 30 & 49.6292 & 76.8323 & 0.921 & Selected \\
2 & 24.14 & 70.82 & 30 & 49.6143 & 76.8517 & 0.921 & \\
3 & 17.59 & 73.62 & 30.0001 & 50.0235 & 76.231 & 0.920 \\
4 & 17.55 & 73.46 & 30 & 50.0168 & 76.2395 & 0.920 \\
5 & 17.62 & 73.77 & 30 & 50.0289 & 76.2221 & 0.920 \\
6 & 21.27 & 78.03 & 30.0002 & 49.8719 & 75.3782 & 0.915 \\
7 & 24.20 & 50.00 & 30.0001 & 44.8546 & 69.5445 & 0.847 \\
\hline
\end{tabular}

Table 17 : Statistically selected formulation F10 (10 mg equvalent $w t)$.

\begin{tabular}{ll}
\hline Chemical (mg) & F10 \\
\hline Ibuprofen & 10 \\
Capmul PG 8 & 24.10 \\
Cremophor EL & 71.02 \\
\hline
\end{tabular}

Table 18: Comparison of responses between projected values, optimized values and experimental values.

\begin{tabular}{|c|c|c|c|c|}
\hline 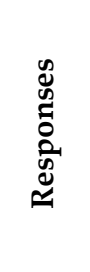 & 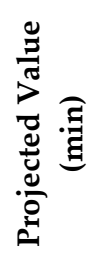 & 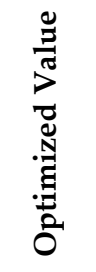 & 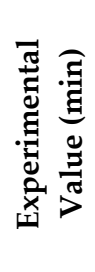 & 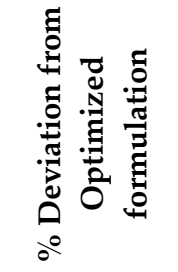 \\
\hline DR5 & 30 & 30 & 27.78 & 7.4 \\
\hline DR15 & 55 & 49.61 & 44.6 & 10.09877041 \\
\hline DR45 & 85 & 76.84 & 74.24 & 3.383654347 \\
\hline
\end{tabular}

emulsifying drug delivery systems (SEDDS) for oral administration with a view to enhance the percent release of Ibuprofen. This Self emulsifying drug delivery system improved dissolution for poorly water soluble drug. Ibuprofen SEDDS were prepared successfully utilizing different proportion of Capmul PG8 and Cremophor EL. \% release of Ibuprofen was determined by using dissolution method. Among the 9 formulation, formulation 6 was showed the highest \% release of drug that was $94.367 \%$. In this formulation amount of active drug was $10 \mathrm{mg}$, $30 \mathrm{mg}$ Capmul PG8 and $70 \mathrm{mg}$ Cremophor EL. A computer based optimization technique, response surface methodology (RSM), was used. Statistically selected formulation F-10 from Diesgn-
Expert 8.0.3.1 Trial Version under experimental study. Experimental study showed quite closer \% release. Here the dependent variables were the amount of Capmul PG8 and Cremophor EL and the independent variables were drug release at 5 minute, 15 minute and 45 minute. To optimize Ibuprofen formulation, Capmul PG8 and Cremophor EL were evaluated at different concentration. The lowest DR5 value was obtained 1.295. Similarly lowest DR15 and DR45 values were 5.965 and 18.816 respectively. The targets for responses were 30\% release for 5 minute, $55 \%$ release for 15 minute, and $85 \%$ release for 45 minute. Thus, this study confirmed that study of SEDDS formulation by using the response surface methodology can be used as a possible alternative to traditional oral formulations of Ibuprofen to improve its \% release.

\section{ACKNOWLEDGEMENT}

The authors are thankful to Department of Pharmacy, University of Asia Pacific, Dhaka, Bangladesh for providing support during this research work.

\section{REFERENCES}

Amidon, G.L., Lennernas, H, Shah, V.P, Crison, J.R. (1995). A theoretical basis for a biopharmaceutic drug classification: the correlation of in-vitro drug product dissolution and in vivo bioavailability. Pharm. Res. 12:413-20. DOI PMid:7617530

Baboota, S., Shakeel, F., Ahuja, A., Ali, J., Shafiq, S. (2007). Design, development and evaluation of novel Nanoemulsion formulations for transdermal potential of celecoxib. Acta. Pharm. 57:315-32. DOI PMid:17878111

Charman, S.A., Charman, W.N., Rogge, M.C., Wilson, T.D., Dutko, F.J. and Pouton, C.W. (1992). Self-emulsifying systems; formulation and biological evaluation of an 
investigative lipophilic compound. Pharm. Res. 9, 87-94. DOI PMid:1589415

Constantinides, P.P. (1995). Lipid micro emulsions for improving drug dissolution and oral absorption: physical and biopharmaceutical aspects. Pharm. Res.: 12: 1561-72. DOI PMid:8592652

Ghorab, M.K., Adeyeye, M.C. (1994). Enhancement of ibuprofen dissolution via wet granulation with betacyclodextrin. Pharm Dev Technol. 6: 305-314. DOI PMid:11485172

Glowka, F.K. (2000). Stereo selective pharmacokinetics of ibuprofen and its lysinate from suppositories in rabbits. Int J Pharm. 199:159-166. DOI

Gursoy, R.M. and Benita, S. (2004). Self emulsifying drug delivery system (SEDDS) for improving oral delivery of lipophilic drugs. Biomed Fharmacother. 58,173-182. DOI

Hauss, D.J. (2007). Oral lipid-based formulations. Adv. Drug Deliv. Rev. 59,667-676. DOI PMid:17618704

Lipinski, C.A. (2002). Poor Aqueous Solubility - an Industry Wide Problem in ADME Screening. American Pharmaceutical Review. 5, 82-85.
Palmer, A.M. (2003). New horizons in drug metabolism, pharmacokinetics and drug discovery. Drug News Perspect. 16,57-62. PMid:12682673

Pouton, C.W. (2000). Lipid formulations for oral administration of drugs: non-emulsifying, self-emulsifying and 'selfmicro emulsifying' drug delivery systems. European Journal of Pharmaceutical Sciences. 11: S93-S98. DOI

Reiss, H. (1975). Entropy-induced dispersion of bulk liquids. J Colloids Interface Sci, 53:61-70. DOI

Debnath, S., Satayanarayana, Kumar, G.V. (2011). Nanoemulsion-a method to improve the solubility of lipophilic drugs. Pharmanest - An International Journal of Advances in Pharmaceutical Sciences, Volume 2(2-3): 72-83.

Tang B., Cheng G., Gu J.C., Xu C.H. (2008). Development of solid self-emulsifying drug delivery systems: preparation techniques and dosage forms. Drug Discovery Today, 13: 606. DOI PMid:18598917 